\title{
Bile cholesterol and viscosity, the keys to discriminating adenomatous polyps from cholesterol polyps by a novel predictive scoring model
}

\author{
Eun Young KIM' ${ }^{1}$, Tae Ho HONG*2
}

'Division of Trauma and Surgical Critical Care, Department of Surgery, The Catholic University of Korea, Seoul St. Mary's Hospital, Seoul, Korea ${ }^{2}$ Division of Hepato-Biliary and Pancreas Surgery, The Catholic University of Korea, Seoul St. Mary's Hospital, Seoul, Korea

Introduction: Adenomatous gallbladder polyps, premalignant lesions of the gallbladder, have fatal outcomes, whereas cholesterol polyps have benign features. Herein, we proposed a novel, predictive scoring model of adenomatous polyps to distinguish them from cholesterol polyps, by analyzing bile components and bile viscosity.

Methods: Patients with gallbladder polyp pathologically confirmed after cholecystectomies were analyzed. After dividing patients into two groups (adenomatous or cholesterol polyps), the clinicopathologic profiles and bile nature, including components and viscosity were compared and a predictive scoring model for adenomatous polyps was assessed.

Results: Eleven adenomatous polyps and 96 cholesterol polyps were analyzed. The variables significantly associated with adenomatous polyps were age $>55$ years (odds ratio $[\mathrm{OR}]=23.550, p=0.020)$, bile viscosity $<7.5$ sec-1 $(\mathrm{OR}=22.539, p=0.012)$, and bile cholesterol $<414.5 \mathrm{mg} / \mathrm{dL}(\mathrm{OR}=10.004, p=0.023)$ and the points for each variable in the predictive scoring model were allocated as 3 , 3 , and 2, respectively. Final scores ranged from 0 to 8 points and the best performance of model at a cutoff of $\geq 6$ points had $90.9 \%$ of sensitivity and $80.2 \%$ of specificity.

Conclusions: Bile viscosity and bile cholesterol accompanied by age were revealed as significant predictors of adenomatous polyps, distinguishing them from cholesterol polyps of gallbladder. It can be the cornerstone for creating accurate guidelines for preoperatively determining treatment strategies of gallbladder polyps. 